\title{
Modulating progenitor accumulation attenuates lung angiogenesis in a mouse model of asthma
}

\author{
T.M. Doyle, R. Ellis, H.J. Park, M.D. Inman and R. Sehmi
}

ABSTRACT: Asthmatic responses are associated with the lung homing of bone marrow (BM)derived progenitors implicated as effectors of disease pathology. Studies have shown that increases in lung extracted vascular endothelial progenitor cells (VEPCs) correlate with airway angiogenesis and declining lung function. We investigated the effect of modulating lung homing of VEPCs on tissue remodelling and airway hyperresponsiveness (AHR).

$B A L B / c$ mice were sensitised to ovalbumin, subjected to a chronic exposure protocol and given early concurrent or delayed treatment with a modulator of progenitor traffic, AMD3100 (CXC chemokine receptor 4 antagonist; inhibits chemotactic activity of stromal-derived factor-1a on VEPCs). After ovalbumin challenge, early haemopoietic stem cells (HSCs) and VEPCs were enumerated along with indices of airway inflammation, lung morphometry and AHR.

Following ovalbumin challenge, there was a decrease in BM and an associated increase in the lung tissue-extracted HSCs and VEPCs, together with increases in airway eosinophilia, microvessel density and AHR. These outcomes were significantly inhibited by early concurrent treatment with AMD3100. Where lung disease was established, delayed treatment with AMD3100 significantly attenuated HSC numbers and lung angiogenesis but only partially reversed sustained AHR compared with untreated ovalbumin-exposed mice.

Progenitor lung homing is associated with the development of asthma pathology, and early modulation of this accumulation can prevent airway remodelling and lung dysfunction.

KEYWORDS: Angiogenesis, asthma, bone marrow progenitors, lung remodelling, stromal-derived factor-1, vascular endothelial progenitor cells

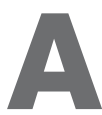
sthma is a chronic inflammatory disease characterised by remodelling of the airways, including goblet cell hyperplasia, thickening of the fibrous region beneath the basement membrane, smooth muscle hyperplasia/hypertrophy and airway angiogenesis [1, 2]. Although inflammation of the airway is an essential component of asthma exacerbations, current anti-inflammatory therapies have been unsuccessful in completely reversing sustained airway hyperresponsiveness (AHR) and this has led to the perspective that remodelling of the lung tissue may have additional functional consequences to those produced by inflammation [3].

It is evident that an association exists between allergen-induced asthmatic responses and mobilisation of haemopoietic progenitor cells (HPCs) from the bone marrow (BM) [4]. These multipotent progenitors, once homed to the lung, can be manipulated by a spectrum of local cytokines and chemokines to differentiate in situ into a variety of inflammatory (eosinophils, basophils and mast cells) [5-7] and/or structural cells (fibrocytes and vascular endothelial cells) [8]. Following allergen challenge in atopic asthmatics [6] and sensitised mice [7], increases in BM-derived HPCs are detected in the lung, which precede the development of lung eosinophilia. In the previous study, SOUTHAм et al. [7] showed that nonlineagecommitted primitive progenitors increased in the lung tissue of mice well after the resolution of airway eosinophilia, suggesting that, in addition to contributing to early inflammatory events, progenitors could be involved in the initiation and/or maintenance of airway structural changes. Phenotypic analysis of primitive lung-extracted progenitors showed a high level of expression for CXC

\section{AFFILIATIONS}

Asthma Research Group, Firestone Institute for Respiratory Health, St Joseph's Healthcare, McMaster University, Hamilton, ON, Canada.

\section{CORRESPONDENCE}

R. Sehmi

Firestone Institute for Respiratory Health

St Joseph's Hospital

Luke Wing

Room L314-6

50 Charlton Avenue East

Hamilton

ON

L8N 4A6

Canada

E-mail: sehmir@mcmaster.ca

Received:

Aug 182010

Accepted after revision:

Oct 112010

First published online:

Nov 112010 
chemokine receptor (CXCR)4, and a corresponding increase in its cognate ligand, stromal-derived factor (SDF)-1 [7, 9]. Since SDF-1 is a potent progenitor chemoattractant that has been shown to be increased in the airways of asthmatics, it may be involved in promoting the lung homing of progenitors in asthma [10-13].

A recent study reported an increase in lung vascular endothelial progenitor cells (VEPCs) following allergen challenge in sensitised mice, which was associated with increased lung angiogenesis, considered to be an early remodelling event in asthma [14, 15]. Interestingly, VEPCs express CXCR4 [13], suggesting that SDF-1 may play a pivotal role in the lung homing of progenitors, specifically VEPCs, to the asthmatic lung. We hypothesise that angiogenesis can be prevented by modulating lung homing of VEPCs within the lung. This was tested in an established chronic allergen exposure mouse model, which characteristically develops both inflammatory and tissue remodelling changes associated with increased AHR [16]. To prevent the migration of VEPCs from the BM to the lung, mice were treated with a CXCR4 antagonist, AMD3100 [17], which has previously been shown to attenuate both eosinophilia and AHR in a mouse model of asthma [18]. In the present study AMD3100, given intranasally was administered either concurrently during the onset of disease or in a reversal/delayed treatment regimen once airway disease was established.

Our data show that localised treatment with concurrent AMD3100 significantly attenuated the accumulation of primitive haemopoietic stem cells (HSCs) and VEPCs, vascularisation of lung tissue, airway eosinophilia, and AHR. In established disease, AMD3100 treatment attenuated accumulation of HSCs and lung vascularisation but only partially reversed sustained AHR. This suggests that progenitor cell increases within the lung are associated with early angiogenic remodelling events and the development of AHR, and modulating progenitor activity or reversing lung angiogenesis can partially resolve previously established airway dysfunction.

\section{MATERIALS AND METHODS \\ Animals}

Female BALB/c mice, 8-10 weeks of age (Charles River Laboratories, Saint-Constant, QC, Canada) were maintained in a pathogen-free environment. All procedures were reviewed and approved by the Animal Research Ethics Board at McMaster University (Hamilton, ON, Canada).

\section{Allergen exposure using the chronic exposure protocol}

Mice ( $n=10$ per group) were sensitised and challenged, as previously described [16] and as shown in figure 1. Outcomes were measured at day 91 (24 h post-ovalbumin), and where mentioned, at day 118 (4 weeks post-ovalbumin).

\section{Treatment intervention}

Mice were given i.n. AMD3100 (Sigma, Mississuaga, ON, Canada), a CXCR4 antagonist [19]. Optimal dosing of the drug $\left(15 \mathrm{mg} \cdot \mathrm{kg}^{-1}\right)$ was established in pilot experiments as the lowest dose capable of preventing allergen-induced increases in airway vascularity in a brief allergen exposure protocol (online supplementary material, fig. S1). Secondary outcome measures for this pilot study included airway inflammation and progenitor cells from lung-extracted tissue. Intervention with AMD3100 consisted of: 1) concurrent treatment with the drug administered $4 \mathrm{~h}$ before each allergen challenge during the 1890 days of exposure (fig. 1a); or 2) reversal treatment with the drug administered on two consecutive days each week for 4 weeks (90-118 days) after cessation of the allergen exposure (fig. 1b). Control mice received ovalbumin plus saline vehicle instead of a drug.

a)

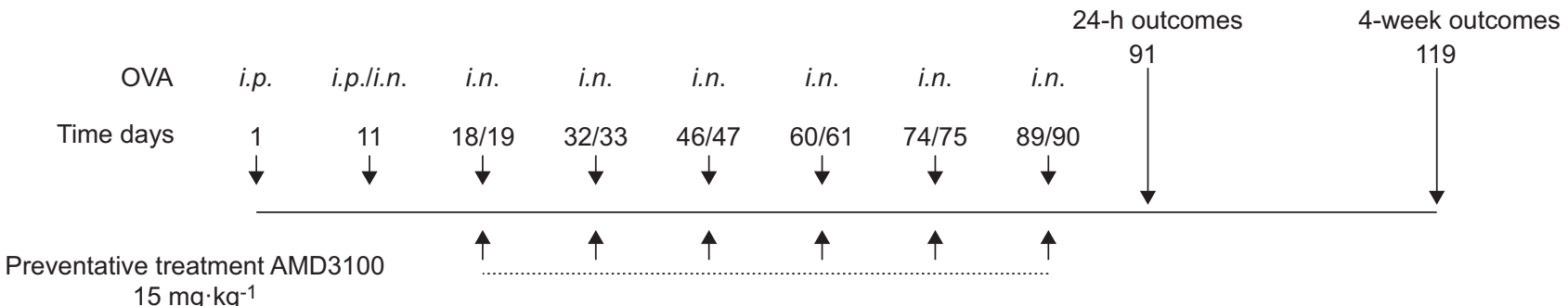

b)

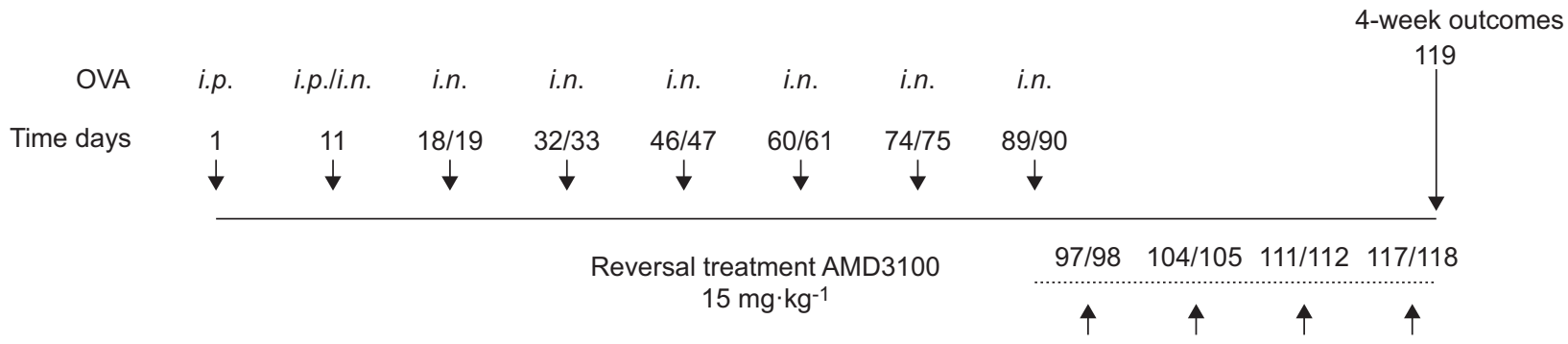

FIGURE 1. Chronic sensitisation and drug treatment protocol. BALB/c mice were sensitised to ovalbumin (OVA; $80 \mu$ in $200 \mu \mathrm{L}$ saline intraperitoneally (i.p.)) on days 1 and 11 and challenged intranasally (i.n.) (100 $\mu \mathrm{g}$ OVA in $25 \mu \mathrm{L}$ saline) on day 11. Mice were then given six 2-day i.n. OVA or saline challenges throughout the 18-90-day period. Drug intervention protocols included a) concurrent or b) reversal regimens. Administration of AMD3100 (15 mg $\left.\mathrm{kg}^{-1}\right)$ or vehicle (i.n.) prior to OVA or saline challenge occurred on the indicated days. Outcomes were measured $24 \mathrm{~h}$ (concurrent outcomes) or 4 weeks (reversal outcomes) after the-final allergen exposure. 

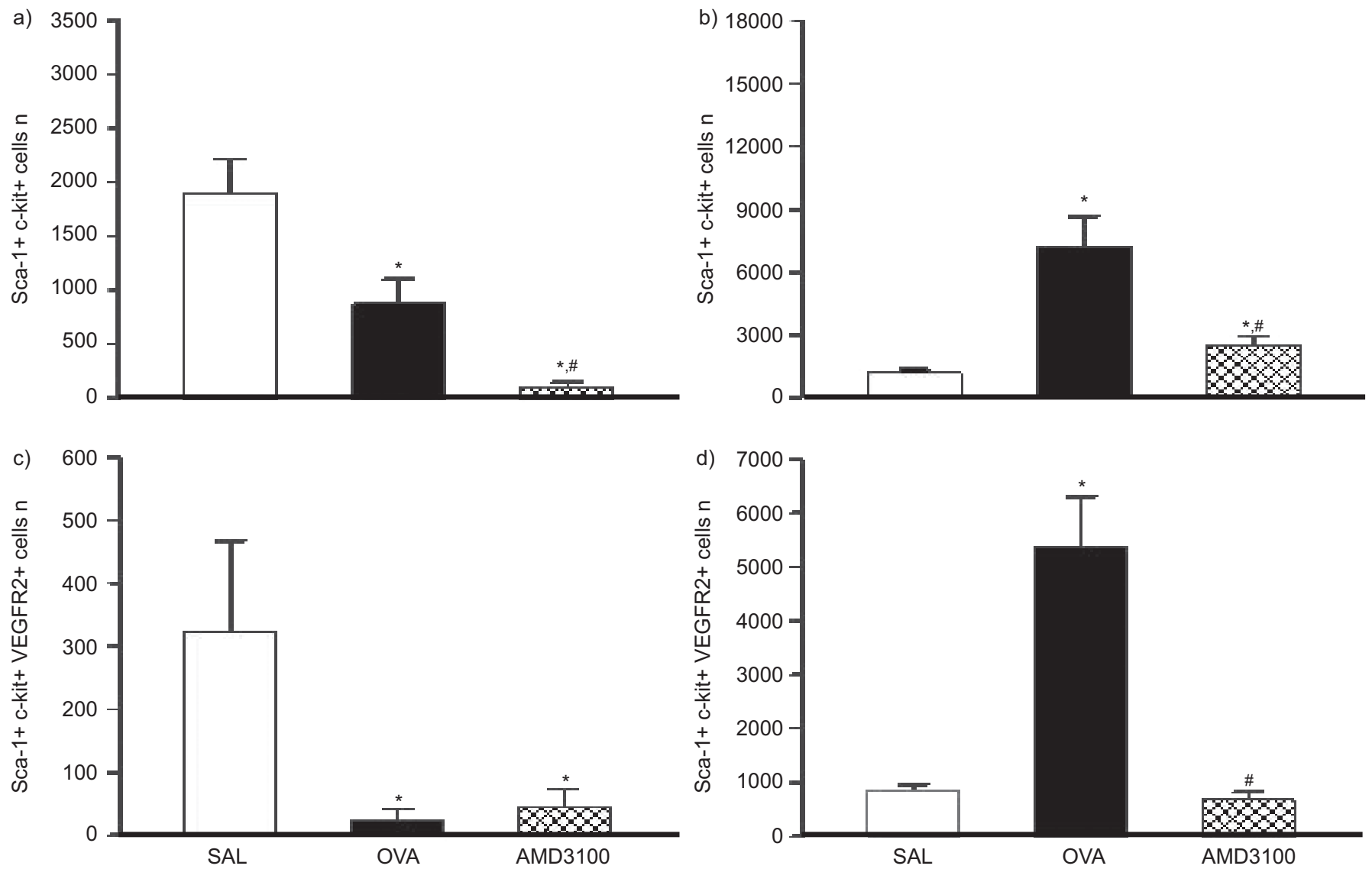

FIGURE 2. Enumeration of $a, b)$ haemopoietic stem cells (Sca1+c-kit+) and c, d) vascular endothelial progenitor cells (Sca-1+c-kit+VEGFR2+) from a, c) bone marrow (BM)- and b, d) lung-extracted cells by flow cytometry. Samples were taken $24 \mathrm{~h}$ after challenge with saline (SAL), allergen (ovalbumin (OVA)) or OVA plus AMD3100 ( $n=10$ mice per group). Compared with SAL, a significant egress from BM and influx into the lungs was detected in the OVA group for all progenitors. Concurrent treatment with AMD3100 further mobilised progenitors from the BM but attenuated the lung homing of these cells. *: $p<0.05$ compared with SAL; ${ }^{*}: p<0.05$ compared with OVA.

\section{Airway responsiveness}

Airway responsiveness to intravenous methacholine was measured based on the response of total respiratory system resistance using the FlexiVent ventilator system (SCIREQ, Montreal, QC, Canada), as previously described [20].

\section{Bronchoalveolar lavage}

Bronchoalveolar lavage (BAL) was performed as previously described [21]. Vascular endothelial growth factor (VEGF) levels in BAL fluid were assessed using a sandwich ELISA kit (R\&D Systems, Minneapolis, MN, USA).

\section{Progenitor cell isolation, immunostaining and flow cytometry}

Mice were sacrificed by exsanguination via cardiac puncture, and lungs were perfused clear of blood with saline and removed from the thoracic cavity, as previously described [7]. Lung tissueassociated cells were extracted from the right lobe by mincing and enzymatic digestion, as previously described [7]. BM samples were flushed from the femur and tibia and mononuclear cells were collected following density gradient centrifugation $(400 \times g$ for $30 \mathrm{~min}$ ) over Accu-Prep ${ }^{\mathrm{TM}}$ (Accurate Chemicals, Westbury, NY, USA). Cells were immunostained with fluorescein isothiocyanateconjugated Sca-1, phycoerythrin-conjugated c-kit (BD Bioscience, Oakville, ON, Canada) and allophycocyanin-conjugated VEGF receptor (VEGFR)2 antibodies (eBioscience Inc., San Diego, CA, USA), or isotype control antibodies $\left(40 \mathrm{~min}\right.$ at $\left.4{ }^{\circ} \mathrm{C}\right)$, and fixed in PBS with 1\% paraformaldehyde (BDH Laboratory Supplies, Mississauga, ON, Canada), and cell data $(100,000$ events in the lymphomononucelar region) was acquired using a FACSCaliber flow cytometer equipped with a 488-nm argon ion laser (BD Instrument Systems, Mississauga, ON, Canada). Primitive progenitor cells (Sca-1+c-kit+) and lineage-committed VEPCs (Sca-1+c-kit+VEGFR2+) were enumerated using the Cellquest software package (BD Biosciences) [14]. Details of data analyses are outlined in the online supplementary material (fig. S2). Absolute numbers of cells were calculated using the percentage of population positivity obtained by flow cytometry and the total white cell counts calculated after separation on Accu-Prep ${ }^{\mathrm{TM}}$.

\section{Lung histology and morphometry}

The left lung lobe was perfused with saline, formalin fixed, embedded in paraffin and cut into $3-\mu \mathrm{m}$ sections. Lung vascularity was identified by staining with polyclonal rabbit anti-human von Willebrand factor (vWF) (Dako, Carpinteria, CA, USA), which cross-reacts with the mouse antigen [22]. Slides were analysed using a customised digital image analysis system (Northern Eclipse; Empix Imaging, Mississauga, ON, Canada). Vessels within a $50-\mu \mathrm{m}$ bandwidth from the main 

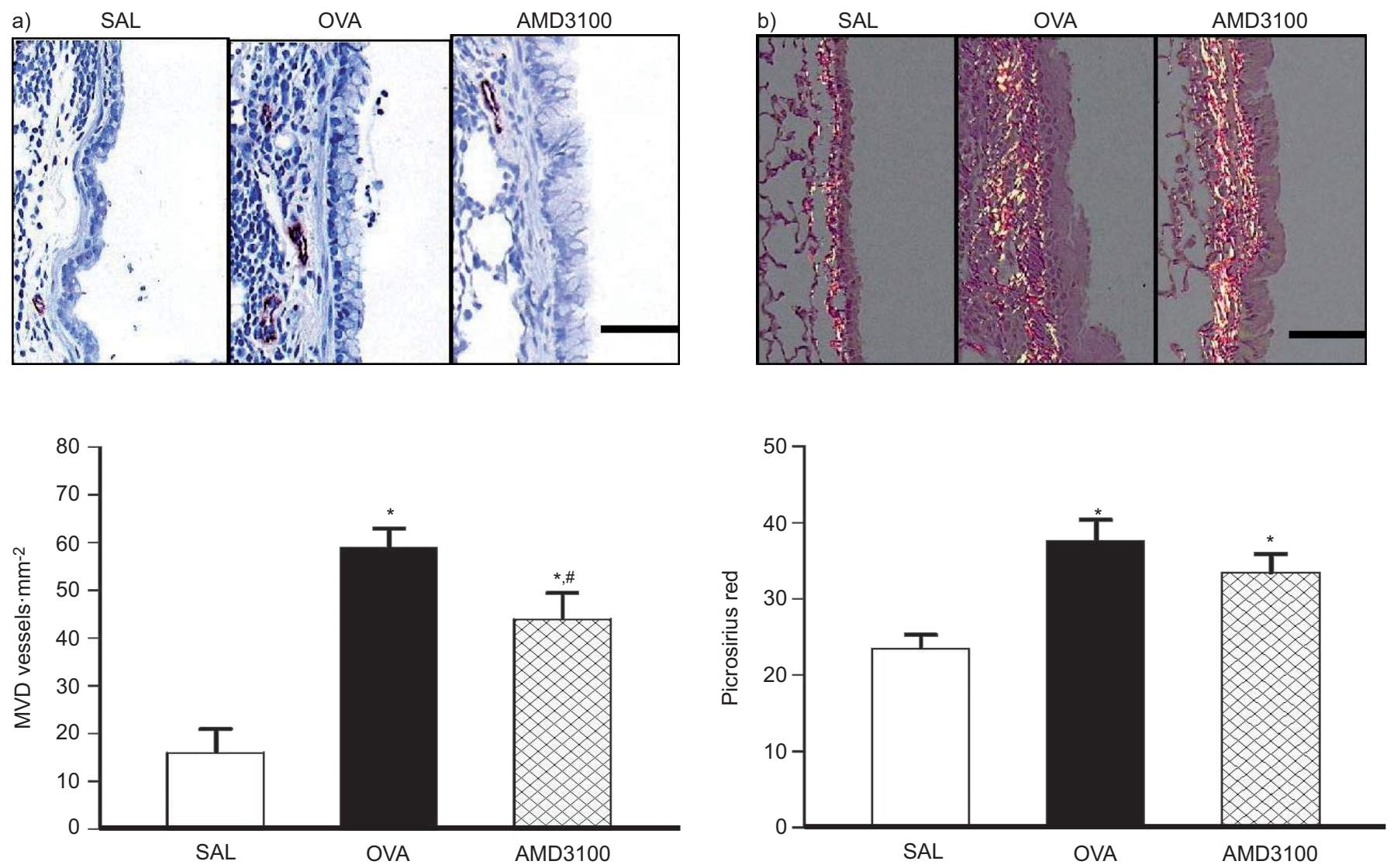

FIGURE 3. a) Microvessel density (MVD) assessed by immunostaining for von Willebrand factor was measured in lung slices taken $24 \mathrm{~h}$ after challenge and b) collagen deposition in lung slices harvested 4 weeks after challenge. Scale bars $=50 \mu \mathrm{m}$. There was a significant increase in both MVD and collagen deposition in the ovalbumin (OVA) group compared with the saline (SAL) group. Concurrent treatment with AMD3100 significantly attenuated MVD but not the level of collagen deposition compared with the OVA group. *: $p<0.05$ compared with SAL; ${ }^{*}: p<0.05$ compared with OVA ( $n=10$ mice per group).

airway that were $\leqslant 10 \mu \mathrm{m}$ in diameter were included in the microvessel quantification. Microvessel density (MVD) was calculated as total positive vessels per bandwidth area.

Lung sections were also stained with picrosirius red antibody for collagen deposition (Clone 1a4; Dako). Tissue was examined within a $20-\mu \mathrm{m}$ peribronchial bandwidth around the main airway and \% positive staining was analysed using Northern Eclipse.

\section{Statistical analysis}

In the text and figure legends, data are presented as mean \pm SEM. Data analysis was performed using STATSISTICA software (Statsoft Inc., Tulsa, OK, USA) to perform ANOVAs; post hoc analyses for between groups comparisons were performed using Duncan's test. $\alpha$ was set at 0.05 .

\section{RESULTS}

Progenitor cells: early concurrent treatment with AMD3100 inhibited allergen-induced lung homing of primitive and vascular endothelial progenitor cells

Using a chronic allergen exposure model in BALB/c mice, early HSCs (Sca1+c-kit+) and lineage-committed VEPCs (Sca-1+c-kit+VEGFR2+) were enumerated in BM and lungextracted cells $24 \mathrm{~h}$ post-challenge with allergen (ovalbumin) or saline (fig. 2). For both HSC and VEPC levels, compared with saline, there was a significant decrease in the BM and a corresponding increase in the lung in the ovalbumin group (fig. $2 \mathrm{a}$ and $\mathrm{b}$ and fig. $2 \mathrm{c}$ and $\mathrm{d}$, respectively).

Intranasal treatment with AMD3100 given concurrently with allergen challenge caused a reduction in BM HSCs compared with the ovalbumin and saline groups, respectively, $24 \mathrm{~h}$ postallergen (fig. 2a). In the lung, concurrent treatment with AMD3100 significantly attenuated the lung homing of HSCs compared with the ovalbumin group (fig. 2b). Although the drug did not cause a significant mobilisation of VEPCs from the BM levels compared with the ovalbumin group (fig. 2c), there was a significant attenuation in the lung homing of VEPCs compared with the ovalbumin group (fig. $2 \mathrm{~d}$ ).

To confirm a selective effect of AMD3100 on progenitor cells, total cell counts of the mononuclear cell population were enumerated from BM- and lung-extracted cell samples. The cell counts showed that in the treatment group, the drug did not significantly lower total cell numbers compared with the ovalbumin group in either of the compartments (online supplementary material; table S1). In addition, the effect of AMD3100 on the directional migrational responses of mouse BM-derived HSCs was demonstrated in vitro. The results showed that AMD3100 selectively attenuated the directional migration of HSCs to SDF-1 but not VEGF in vitro (online supplementary material; table S2). 


\begin{tabular}{|c|c|c|c|}
\hline \multirow[t]{2}{*}{ TABLE 1} & \multirow[b]{2}{*}{ Saline } & $\begin{array}{l}\text { ell counts in } \\
\text { samples }\end{array}$ & \multirow[b]{2}{*}{$\begin{array}{l}\text { Ovalbumin plus } \\
\text { AMD3100 }\end{array}$} \\
\hline & & Ovalbumin & \\
\hline TCC $\times 10^{2}$ cells & $840 \pm 128$ & $1840 \pm 144^{*}$ & $917 \pm 120^{\#}$ \\
\hline Neutrophils cells & $13 \pm 3$ & $251 \pm 9^{*}$ & $57 \pm 9^{*, \#}$ \\
\hline Lymphocytes cells & $140 \pm 23$ & $502 \pm 213^{*}$ & $57 \pm 21^{*}$ \\
\hline Eosinophils $\times 10^{2}$ cells & $6 \pm 1$ & $493 \pm 124^{*}$ & $14 \pm 4^{\star, \#}$ \\
\hline Macrophages $\times 10^{2}$ cells & $702 \pm 97$ & $1333 \pm 286^{*}$ & $820 \pm 32^{\#}$ \\
\hline \multicolumn{4}{|c|}{$\begin{array}{l}\text { Data are presented as mean } \pm \text { SEM. TCC: total cell count. Cells were counted in } \\
\text { bronchoalveolar lavage samples collected from mice ( } n=10 \text { per group) } \\
\text { sensitised by the chronic exposure protocol with concurrent treatment with a } \\
\text { drug. Measurements were made at } 24 \mathrm{~h} \text { after saline, ovalbumin or ovalbumin } \\
\text { plus AMD3100 challenge. }{ }^{*}: p<0.05 \text { compared with saline; }{ }^{*}: p<0.05 \\
\text { compared with ovalbumin. }\end{array}$} \\
\hline
\end{tabular}

Airway remodelling: concurrent treatment with AMD3100 significantly attenuated allergen-induced airway angiogenesis but not collagen deposition

Formation of new blood vessels was assessed by staining for vWF in mouse lung slices (fig. 3a). Enumeration of MVD showed a significant increase in the ovalbumin group compared with the saline group, $24 \mathrm{~h}$ post-allergen. Concurrent treatment with AMD3100 significantly attenuated MVD levels when compared with the ovalbumin group but were not completely reversed as they remained significantly higher than for the saline group.

Collagen deposition, as a marker of airway fibrosis, was detected by staining with picrosirius red (fig. 3b). The level of collagen deposition was assessed at 4 weeks post-challenge as this has been previously shown to be the optimal time point for detecting allergen-induced airway fibrosis (the cellular inflammation at earlier time points interferes with the accurate immunostaining for fibrosis) [16]. In the ovalbumin group, collagen deposition was significantly elevated when compared with the saline group. Concurrent drug treatment had no effect on collagen levels compared with the ovalbumin group and these levels were significantly greater than in the saline group (fig. 3b).

\section{Airway inflammation: concurrent treatment with AMD3100 significantly attenuated allergen-induced airway inflammation}

There was a significant increase in inflammatory cells in the BAL including eosinophils in the ovalbumin compared with the saline group $24 \mathrm{~h}$ after allergen challenge (table 1). Mice treated concurrently with AMD3100 showed a significant attenuation in total cells including neutrophils, eosinophils and macrophages compared with the ovalbumin group (table 1).

\section{Airway responsiveness: concurrent treatment with AMD3100 significantly attenuated allergen-induced airway hyperresponsiveness}

Airway responsiveness was assessed by measuring the maximum respiratory system resistance to incremental doses of i.v. methacholine (fig. 4a). A significant increase in maximum
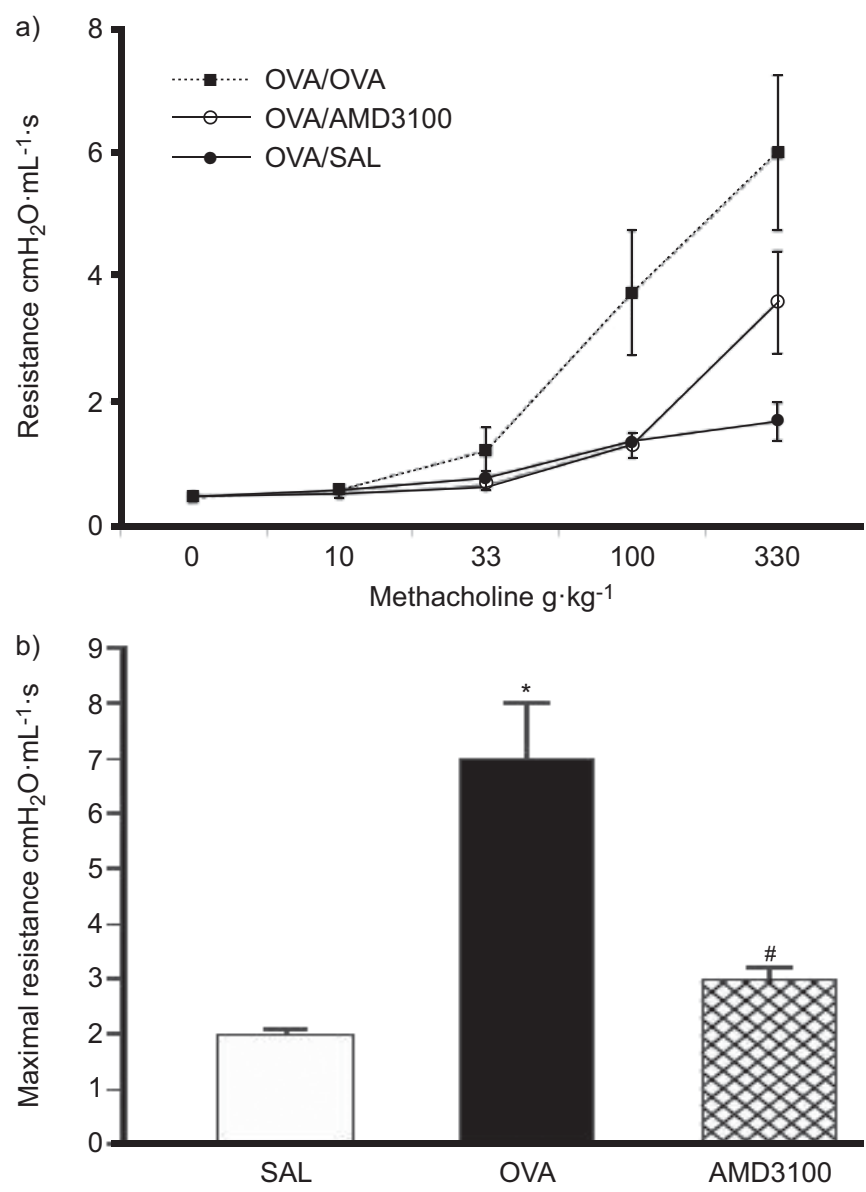

FIGURE 4. Airway responses were measured as a) maximum resistance to intravenous methacholine in mice $24 \mathrm{~h}$ after challenge ( $\mathrm{n}=10$ mice per group). b) Maximum resistance in response to $100 \mathrm{~g} \cdot \mathrm{kg}^{-1}$ methacholine i.v. * $p<0.05$ compared with saline (SAL); \#: $p<0.05$ compared with ovalbumin (OVA). There was a significant increase in airway hyperresponsiveness in the OVA group compared with the SAL group and this was significantly attenuated by concurrent treatment with AMD3100.

resistance to methacholine was observed $24 \mathrm{~h}$ post-allergen in the ovalbumin compared with the saline group. In the drug treatment group, there was a significant attenuation in resistance compared with the ovalbumin group (fig. $4 b$ ).

\section{Reversal treatment regimen}

We studied the effect of modulating progenitor cell activity in a model where indices of allergic airway disease were already established. The effect of modulating progenitor cell activity was investigated during a 4-week period following 90 days of allergen challenge, at which time point it has previously been shown that despite the subsidence of inflammation, airway eosinophilia, airway remodelling and AHR persist [16, 23].

Progenitor cells: delayed treatment with AMD3100 inhibited allergen-induced lung homing of primitive progenitor cells Following the reversal treatment regimen with AMD3100 (fig. 1b), HSCs and VEPCs were enumerated 4 weeks postchallenge (fig. 5). Within the BM, compared with saline, the absolute numbers of HSCs were significantly lower in the ovalbumin group and drug treatment did not further reduce 

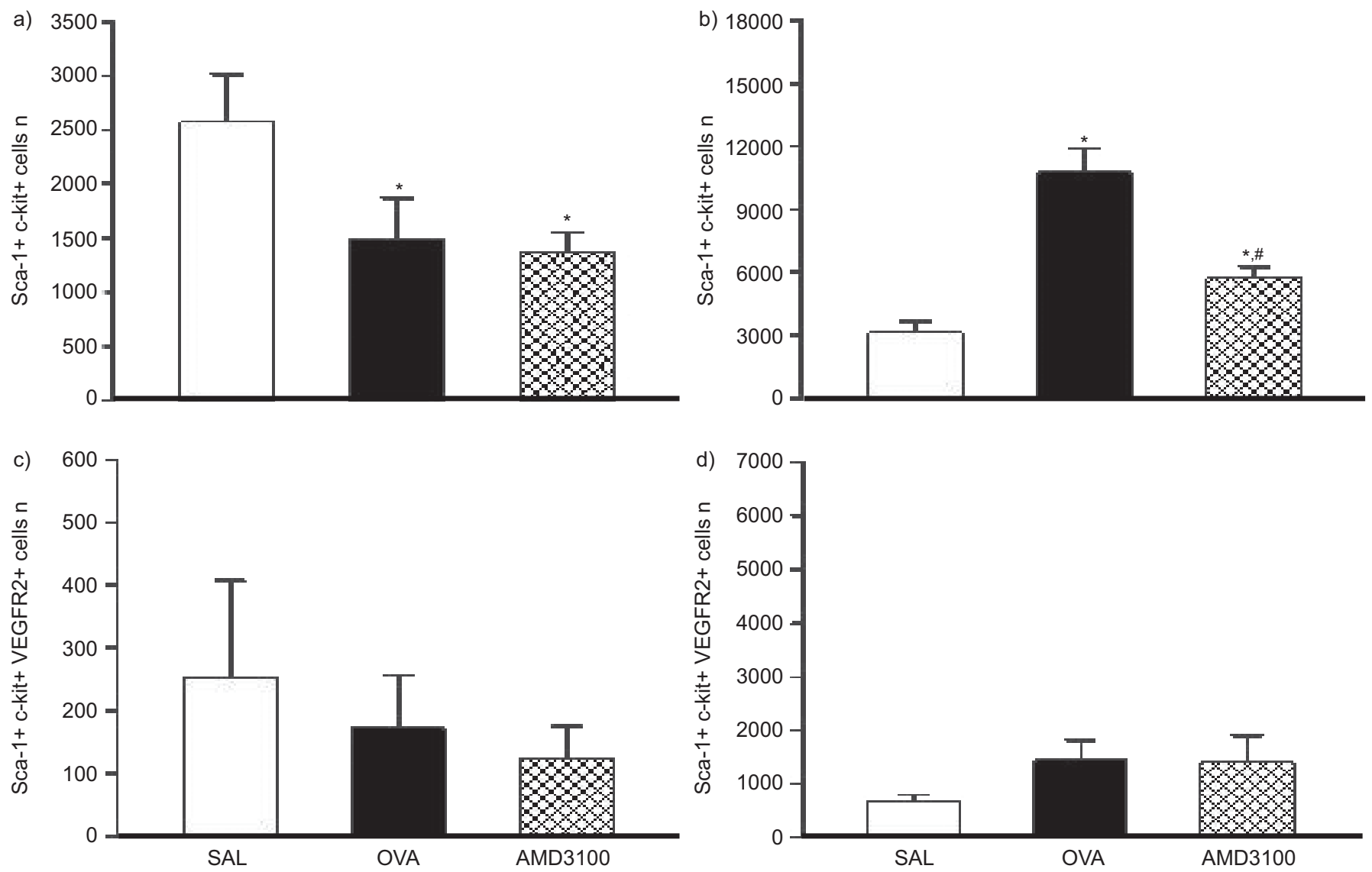

FIGURE 5. Enumeration of progenitors in $a, c)$ bone marrow (BM)-extracted and b, d) lung-extracted cell samples. Using the reversal treatment regimen, a, b) primitive haemopoietic stem cells (Sca1+c-Kit+) and c, d) vascular endothelial progenitor cells (Sca1+c-Kit+VEGFR2+) were measured by flow cytometry 4 weeks after challenge. *: $p<0.05$ compared with saline (SAL); ${ }^{*}: p<0.05$ compared with ovalbumin (OVA) ( $n=10$ mice per group).

these numbers (fig. 5a). Within the lung, HSC numbers remained higher in the ovalbumin group compared with the saline group 4 weeks post-allergen, and delayed treatment with AMD3100 significantly reduced these levels compared with untreated ovalbumin (fig. 5b).

Conversely, in the VEPC populations in both the BM and the lung, compared with saline, there was no significant difference in the ovalbumin group and treatment with AMD3100 during the 4 weeks after challenge did not affect these levels (fig. $5 \mathrm{c}$ and $\mathrm{d})$.

\section{Tissue remodelling and airway function: delayed treatment with AMD3100 significantly attenuated allergen-induced airway angiogenesis}

Significantly greater levels of vWF staining were found in the ovalbumin group compared with the saline group 4 weeks after allergen challenge (fig. 6a) and drug treatment significantly reduced MVD levels compared with the ovalbumin group.

Collagen deposition in the ovalbumin-exposed mice was significantly greater than in the saline group 4 weeks postchallenge. Drug treatment had no effect on fibrosis levels compared with the ovalbumin group and these levels remained significantly greater than in the saline group.
Corresponding to primitive progenitor cell numbers in the lung and measures of lung vascularity, there was a sustained increase in airway resistance in the ovalbumin group 4 weeks after allergen challenge (fig. 6c). Delayed treatment with AMD3100 reduced methacholine airway resistance to levels comparable with the saline group but not significantly lower than the ovalbumin group $(p=0.054)$.

\section{BAL VEGF levels: allergen-induced increases in BAL VEGF} levels were not affected by treatment with AMD3100

A significant increase in VEGF levels was detected in the ovalbumin compared with the saline group $24 \mathrm{~h}$ post-allergen challenge and drug treatment had no effect (table 2). In the reversal treatment group, BAL VEGF levels measured 4 weeks after allergen challenge in the ovalbumin group and drug treatment groups had returned to saline levels (table 2).

\section{DISCUSSION}

Asthma has a systemic component involving the mobilisation and lung homing of BM-derived progenitor cells that may contribute to inflammation and tissue remodelling, including angiogenesis [4, 15]. We hypothesise that modulating lung homing of VEPCs can prevent the increased bronchial vascularisation and, in turn, modulate lung function in a mouse model of asthma. Our data show that: 1) in allergic inflammatory responses, both primitive and lineage-committed progenitors 

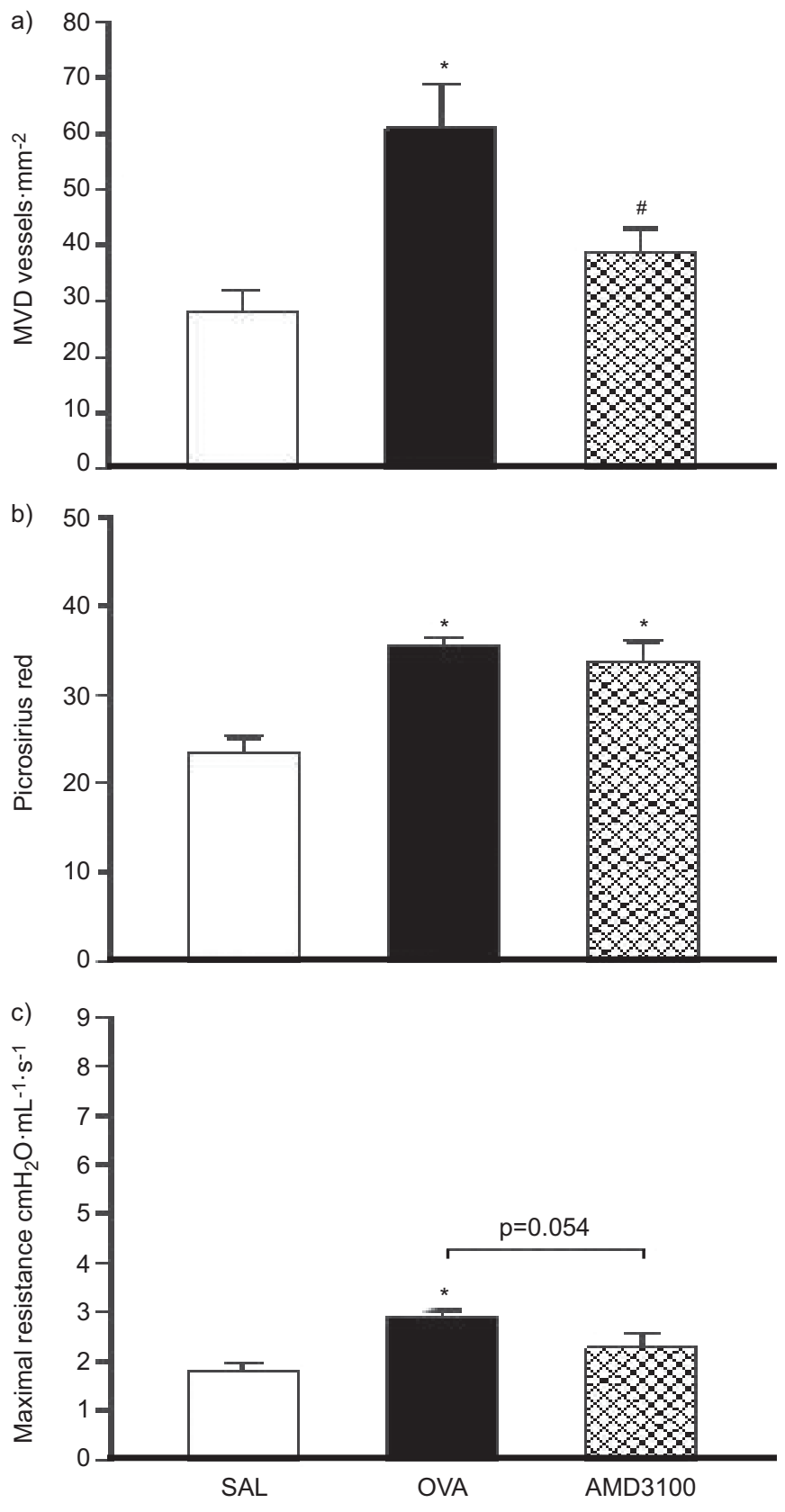

FIGURE 6. Effect of modulating progenitor cell traffic on reversing established markers of airway remodelling. a) Microvessel density (MVD), b) collagen deposition and c) airway resistance in response to methacholine. Outcomes were measured in mice from the reversal treatment regimen at 4 weeks (day 118) after challenge in the saline (SAL), allergen (ovalbumin (OVA)) or OVA plus AMD3100 groups ( $n=8-10$ mice per group). Compared with SAL, there was a sustained increase in airway hyperresponsiveness in the OVA group, which was reduced to SAL levels following delayed treatment with AMD3100. *: $p<0.05$ compared with SAL; ${ }^{\#}: p<0.05$ compared with OVA.

migrate from the BM to the lung; 2) modulating progenitor cell accumulation during the early onset of disease prevents increased vascularisation of airway tissue and the development of AHR; and 3) in an established disease, treatment with AMD3100-attenuated HSC levels in the lung and airway vascularity, and only partially resolved sustained AHR.

\begin{tabular}{|c|c|c|c|c|}
\hline \multirow[t]{2}{*}{ TABLE 2} & \multicolumn{4}{|c|}{$\begin{array}{l}\text { Levels of vascular endothelial growth factor } \\
\text { (VEGF) determined in bronchoalveolar lavage } \\
\text { (BAL) samples by ELISA }\end{array}$} \\
\hline & & Saline & Ovalbumin & $\begin{array}{l}\text { Ovalbumin plus } \\
\text { AMD3100 }\end{array}$ \\
\hline \multirow{2}{*}{\multicolumn{2}{|c|}{$\begin{array}{l}\text { Concurrent treatment } \\
\text { Reversal treatment }\end{array}$}} & $59.01 \pm 4.19$ & $105.1 \pm 11.9^{\star}$ & $134.0 \pm 14.4^{*}$ \\
\hline & & $58.39 \pm 3.9$ & $61.9 \pm 2.9$ & $77.14 \pm 8.6^{\star}$ \\
\hline \multicolumn{5}{|c|}{$\begin{array}{l}\text { Data are presented as mean } \pm \text { SEM. Levels of VEGF }\left(\mathrm{pg} \cdot \mathrm{mL}^{-1} \text { ) were determined in }\right. \\
\text { BAL samples ( } \mathrm{n}=10 \text { mice per group) by ELISA at } 24 \mathrm{~h} \text { post-challenge in the } \\
\text { concurrent (early) treatment group and } 4 \text { weeks post-challenge in the reversal } \\
\text { (delayed) treatment group. Treatment with AMD } 3100 \text { had no significant effect on } \\
\text { VEGF levels compared with the ovalbumin group. } *: p<0.05 \text { compared with saline. }\end{array}$} \\
\hline
\end{tabular}

Our data showed a rapid and sustained allergen-induced lung homing of VEPCs, which correlated with the level of lung vascularity $\left(r^{2}=0.46 ; p<0.005\right)$ and airway resistance $\left(r^{2}=0.71\right.$, $\mathrm{p}<0.005)$ in a mouse model of asthma, consistent with previous studies [14, 24]. Lung-extracted VEPCs can differentiate into endothelial cells with the capacity to form tube-like structures resembling vasculature $[25,26]$. In this study, levels of lung-extracted VEPCs were elevated in the lungs of allergen-challenged mice at $24 \mathrm{~h}$ post-ovalbumin challenge but not 4 weeks post-ovalbumin (fig. $2 \mathrm{~d}$ and fig. $5 \mathrm{~d}$ ), whilst primitive progenitor cells and vascularity were increased $24 \mathrm{~h}$ post-ovalbumin (fig. $2 \mathrm{~b}$ and fig. $3 \mathrm{a}$ ) and remained elevated up to 4 weeks post-ovalbumin challenge compared with saline (fig. $5 \mathrm{~b}$ and fig. 6a). This suggests that homing and rapid terminal differentiation of lung-extracted VEPCs is limited to the early onset of the disease and during an active inflammatory process. The sustained increase in lung levels of HSCs suggest that these cells may be involved in maintaining established tissue remodelling and contributing to a priming mechanism for future inflammatory responses [27]. Studies of tumour vasculature have also shown that whilst BM-derived VEPCs contribute to the early angiogenic switch, once the tumour is established, the traffic and migration of these cells is limited and extramedullary processes perpetuate the vascularisation of the tumour $[25,28]$.

Recent studies have shown that despite the capacity of lungextracted VEPCs to form tube-like structures in culture, few green fluorescent protein-labelled VEPCs actually incorporate into the vascular structures within the lung following allergen challenge in sensitised mice [14, 25, 29]. Instead, these progenitors home to perivascular sites and secrete pro-inflammatory and proangiogenic factors that have the potential to orchestrate the inflammatory response and tissue remodelling changes associated with asthma $[24,30]$. Regardless of the exact role of these cells, our study shows that that modulation of lung homing of BM-derived progenitor cells during disease onset attenuated lung vascularity and the subsequent development of airway inflammation and AHR [2].

In this study, AMD3100 was administered i.n. to focus its effect on the lung. Despite the localised delivery, AMD3100 had a systemic effect, seen as increased mobilisation of primitive progenitors from the $\mathrm{BM}$ probably due to drug-mediated 
destabilisation of the SDF-1/CXCR4 axis within the stroma [31]. Analyses of the target organ, however, showed that AMD3100 given by the concurrent treatment regimen inhibited the lung homing of both HSCs and VEPCs in response to allergen challenge. Treatment with AMD3100 also significantly prevented airway angiogenesis, BAL eosinophilia and AHR, supporting the proposal that by preventing progenitor cell lung homing, precursors for new blood vessels were attenuated and, thus, increased lung vascularisation was inhibited. Reduced vascular activity could, in turn, attenuate oedema formation and the resultant airway thickening, and could be manifested as a decrease in airway resistance [32].

In the reversal treatment regimen, AMD3100 showed a reduction in the total lung-extracted HSCs only, and an associated reversal in the level of airway vascularisation and sustained AHR. It is probable that longer duration of treatment and higher doses of AMD3100 may have attenuated all these outcomes to a greater extent. Interestingly, since these results were taken once the inflammation (airway eosinophilia) had subsided, these data indicate that the ability of AMD3100 to decrease angiogenesis and AHR may be independent of its anti-inflammatory properties.

A second receptor for SDF-1, CXCR7, has recently been discovered [33] and although signalling through this new receptor is still controversial, recent studies suggest that decoy and scavenger activity of CXCR7 might be important for the fine tuning of the mobility of progenitors in the BM and in the lymphoid organs [34]. The scavenger activity of CXCR7 towards SDF-1 is thought to generate guidance cues for CXCR4-dependent migration. In the adult mammal, CXCR7 appears to be strongly expressed in many tumours, being seen on the emerging neovasculature and tumour cells where it appears to function by blocking apoptosis and, thus, allowing cellular proliferation [35]. Although the current study did not target this second receptor for SDF-1, the contribution of this receptor to SDF-1-mediated VEPC lung accumulation and angiogenesis following allergen challenge may explain why the delayed treatment with AMD3100 did not completely reverse the level of lung extracted HSC, tissue vascularity or sustained AHR.

Despite an attenuation of progenitor cell influx and angiogenesis, collagen deposition remained unchanged. Since fibrocytes express CXCR4 [36], it was postulated that treatment with AMD3100 would attenuate airway fibrosis. It is possible that other compensatory mechanisms may have responded to allergen challenge despite the ability of AMD3100 to potentially prevent lung homing of fibrocytes, although this was not measured in the current study. The activation of local fibroblasts to differentiate into myofibroblasts or the transdifferentiation of epithelial cells may have contributed to increased collagen deposition in the absence of a systemic component [8]. A multi-pronged approach to treating tissue remodelling that reverses collagen deposition may be an additional therapeutic target for the optimal treatment of airway dysfunction in asthma.

VEGF is a potent angiogenic factor found in BAL of asthmatics and capable of stimulating increased lung neoangiogenesis through local overexpression [37]. In the ovalbumin group, increased levels of BAL VEGF were detected $24 \mathrm{~h}$ postchallenge, which returned to saline levels in the following 4 weeks post-challenge. This is contrary to the level of MVD, which was increased $24 \mathrm{~h}$ post-challenge in the ovalbumin group and was maintained even 4 weeks post-challenge. It has been proposed that a balance between pro- and anti-angiogenic factors controls the local level of angiogenesis. As antiangiogenic factors, such as angiopoietin, were not measured in this study, it remains unclear as to whether an imbalance between the pro- and anti-angiogenic factors permitted lower levels of VEGF to maintain increased vascularisation of the lung tissue.

In summary, we report that during allergic airway disease onset, modulating progenitor cell accumulation in the lung prevents airway angiogenesis and the development of AHR. Furthermore, in established disease, this treatment reversed indices of airway angiogenesis but only partially resolved sustained AHR. These findings suggest that during early onset of asthma, modulating progenitor cell homing to the lung may be a viable therapy for preventing the development of airway dysfunction but that in established disease this therapy alone may not be effective.

\section{SUPPORT STATEMENT}

This study was supported by grants from the Canadian Institutes of Health Research and GlaxoSmithKline-CIRF.

\section{STATEMENT OF INTEREST}

A statement of interest for the study itself can be found at www.erj ersjournals.com/site/misc/statements.xhtml

\section{ACKNOWLEDGEMENTS}

The authors are grateful to I.M. Babirad and K. Peng (Asthma Research Group, Firestone Institute for Respiratory Health, St Joseph's Healthcarem McMaster University, Hamilton, ON, Canada) for their technical contributions to this study.

\section{REFERENCES}

1 Jeffery PK. Remodeling in asthma and chronic obstructive lung disease. Am J Respir Crit Care Med 2001; 164: S28-S38.

2 Wilson JW, Hii S. The importance of the airway microvasculature in asthma. Curr Opin Allergy Clin Immunol 2006; 6: 51-55.

3 Pascual RM, Peters SP. Airway remodeling contributes to the progressive loss of lung function in asthma: an overview. J Allergy Clin Immunol 2005; 116: 477-486.

4 Gauvreau GM, Denburg JA. Hemopoietic progenitors: the role of eosinophil/ basophil progenitors in allergic airway inflammation. Expert Rev Clin Immunol 2005; 1: 87-101.

5 Kim YK, Uno M, Hamilos DL, et al. Immunolocalization of CD34 in nasal polyposis. Effect of topical corticosteroids. Am J Respir Cell Mol Biol 1999; 20: 388-397.

6 Dorman SC, Efthimiadis A, Babirad I, et al. Sputum CD34 $4^{+} \mathrm{IL}-5 \mathrm{Ra}^{+}$ cells increase in sputum after allergen: evidence for in situ eosinophilopoiesis. Am J Respir Crit Care Med 2003; 169: 573-577.

7 Southam DS, Widmer N, Ellis R, et al. Increased eosinophil-lineage committed progenitor cells in the lung of allergen challenged mice. J Allergy Clin Immunol 2005; 115: 95-102.

8 Schmidt M, Sun G, Stacey MA, et al. Identification of circulating fibrocytes as precursors of bronchial myofibroblasts in asthma. J Immunol 2003; 170: 380-389. 
9 Sergejeva S, Malmhäll C, Lotväll J, et al. Increased numbers of $\mathrm{CD}^{2} 4^{+}$cells in nasal mucosa of allergic rhinitis patients: inhibition by a local steroid. Clin Exper Allergy 2005; 35: 34-38.

10 Dorman SC, Babirad I, Post J, et al. Progenitor egress from the bone marrow after allergen challenge: role of stromal cell-derived factor $1 \alpha$ and eotaxin. J Allergy Clin Immunol 2005; 115: 501-507.

11 Catalli AE, Thomson JV, Babirad IM, et al. Modulation of $\beta 1-$ integrins on hemopoietic progenitor cells after allergen challenge in asthmatic subjects. J Allergy Clin Immunol 2008; 122: 803-810.

12 Sehmi R, Thomson JV, Catalli AE. Allergy and the bone marrow: transmigration pathways of hemopoietic progenitor cells from the bone marrow. In: Pawankar R, Holgate S, Rosenwasser LJ, eds. Allergy Frontiers: Classification and Pathomechanisms. Tokyo, Springer, 2009; pp. 421-432.

13 Tilling L, Chowienczyk P, Clapp B. Progenitors in motion: mechnisms of mobilization of endothelial progenitor cells. $\mathrm{Br} J$ Clin Pharmacol 2009; 68: 484-492.

14 Asosingh K, Swaidani S, Aronica M, et al. Th1- and Th2-dependent endothelial progenitor cell recruitment and angiogenic switch in asthma. J Immunol 2007; 178: 6482-6494.

15 Asosingh K, Erzurum SC. Angioplasty in asthma. Biochem Soc Trans 2009; 37: 805-810.

16 Leigh R, Ellis R, Wattie J, et al. Dysfunction and remodeling of the mouse airway persist after resolution of acute allergen-induced airway inflammation. Am J Respir Cell Mol Biol 2002; 27: 526-535.

17 Broxmeyer HE, Hangoc G, Cooper S, et al. AMD3100 and CD26 modulate mobilization, engraftment, and survival of hematopoietic stem and progenitor cells mediated by the SDF-1/CXCL12CXCR4 axis. Ann N Y Acad Sci 2007; 1106: 1-19.

18 Lukacs NW, Berlin A, Schols D, et al. AMD3100, a CXCR4 antagonist, attenuates allergic lung inflammation and airway hyperreactivity. Am J Pathol 2002; 160: 1353-1360.

19 Hatse S, Princen K, Bridger G, et al. Chemokine receptor inhibition by AMD3100 is strictly confined to CXCR4. FEBS Lett 2002; 527: 255-262.

20 Southam DS, Ellis R, Wattie J, et al. Components of airway hyperresponsiveness and their associations with inflammation and remodeling in mice. J Allergy Clin Immunol 2007; 119: 848-854.

21 Inman MD, Ellis R, Wattie R, et al. Allergen-induced increases in airway responsiveness, airway eosinophilia and bone marrow eosinophil progenitors in mice. Am J Respir Crit Care Med 1999; 21: 473-479.

22 Pusztaszeri MP, Seelentag W, Bosman FT. Immunohisochemical expression of endothelial markers CD31, CD34 von Willebrand
Factor, and Fli-1 in normal human tissues. J Histochem Cytochem 2006; 54: 385-395.

23 Leigh R, Ellis R, Wattie J, et al. Type 2 cytokines in the pathogenesis of sustained airway dysfunction and airway remodeling in mice. Am J Respir Crit Care Med 2004; 169: 860-867.

24 Asosingh $\mathrm{K}$, Hanson J, Cheng G, et al. Allergen-induced eotaxin-rich, angiogenic bone marrow progenitors: a blood-borne cellular envoy for lung eosinophilia. J Allergy Clin Immunol 2010; 125: 918-925.

25 Larrivee B, Niessen K, Pollet I, et al. Minimal contribution of marrow-derived endothelial precursors to tumor vasculature. Journal Immunol 2005; 175: 2890-2899.

26 Jones CP, Pitchford SC, Lloyd CM, et al. CXCR2 mediates the recruitment of endothelial progenitor cells during allergic airways remodeling. Stem Cells, 2009: 1-15.

27 Walters EH, Soltani A, Reid D, et al. Vascular remodelling in asthma. Curr Opin Allergy Clin Immunol 2008; 8: 39-43.

28 Gao DD, Nolan DJ, Mellick AS, et al. Endothelial progenitor cells control the angiogenic switch in mouse lung metastasis. Science 2008; 319: 195-198.

29 Khakoo AY, Finkel T. Endothelial progenitor cells. Annu Rev Med 2005; 56: 79-101.

30 Allakhverdi Z, Comeau MR, Smith DE, et al. CD34+ hemopoietic progenitor cells are potent effectors of allergic inflammation. J Allergy Clin Immunol 2009; 123: 472-478.

31 Broxmeyer HE, Orschell CM, Clapp DW, et al. Rapid mobilization of murine and human hematopoietic stem and progenitor cells with AMD3100, a CXCR4 antagonist. J Exp Med 2005; 201: 1307-1318.

32 Paredi P, Barnes PJ. The airway vasculature: recent advances and clinical implications. Thorax 2009; 64: 444-450.

33 Balabanian K, Lagane B, Infantiano S, et al. The chemokine SDF-1/ CXCL12 binds to and signals through the orphan receptor RDCI in T lymphocytes. J Biol Chem 2005; 280: 35760-35766.

34 Burns JM, Summers BC, Wang Y, et al. A novel chemokine receptor for SDF-1 and I-TAC involved in cell survival, cell adhesion and tumor development. J Exp Med 2006; 203: 2201-2213.

35 Thelen M, Theocharis S. CXCR7, CXCR4 and CXCL12: an eccentric trio? J Neuroimmunol 2008; 198: 9-13.

36 Phillips RJ, Burdick MD, Hong K, et al. Circulating fibrocytes traffic to the lungs in reponse to CXCL12 and mediate fibrosis. J Clin Invest 2004; 114: 438-446.

37 Simcock DE, Kanabar V, Clarke GW, et al. Proangiogenic activity in bronchoalveolar lavage fluid from patients with asthma. Am J Respir Crit Care Med 2007; 176: 146-153. 